\title{
Squeezing out the catalysts: the disulphide bond exchange in aryl di- sulphides at high hydrostatic pressure
}

\author{
Szymon Sobczak, * Paulina Ratajczyk and Andrzej Katrusiak* \\ Faculty of Chemistry, Adam Mickiewicz University, Uniwersytetu Poznańskiego 8, 61-614 Poznań, Poland \\ KEYWORDS high-pressure chemistry, disulphide exchange reaction, green chemistry, catalyst-free process
}

\begin{abstract}
Exchange of the disulphide bond is a prominent example of equilibrium reactions. Therefore, the wide library of disulphide exchange reactions requires the application of catalysts, such as reducing agents, strong bases, ultraviolet light, or ultrasounds to stimulate higher conversion yields. The recent observation of pressure-induced exchange of aryl disulphides in the absence of catalysts or other stimuli prompted our study on the mechanism explaining the effect of high pressure on the reaction equilibrium. We employed the pressure between 100 and $400 \mathrm{MPa}$, for promoting the exchange reactions between various homodimeric aryl disulphides and for optimized conditions obtained $100 \%$ yields in pure singlecrystal form of the heterodimer. The reactions were performed in a diamond-anvil cell, as well as in a hydraulic piston-andcylinder press, and the products were characterized by X-ray diffraction, mass and NMR spectroscopy. The prominent role of high-entropy states, not attainable at ambient conditions, as well as the high-entropy nucleation, kinetic crystallization and other effects associated with the high-pressure environment, is apparent. This entropy-driven reactions are an efficient, environmentally-friendly, one-pot method for obtaining pure crystalline heterodimeric disulphides.
\end{abstract}

\section{INTROODUCTION}

Aryl disulphides find versatile applications owing to the properties of the $\mathrm{C}-\mathrm{S}$ bond. This is in the coatings of metal surfaces, anticorrosive agents, paints, oils, and lubricants ${ }^{1-3}$ as well as in active pharmaceuticals ingredients (e.g. in Lansoprazole, Sulindac, Esomeprazole, and Quetiapine) used to treat cancer, inflammation, asthma, Alzheimer, Parkinson, HIV and other diseases. ${ }^{4-10}$ Reversible exchange of disulphide bonds underlies living functions, contributing to the stability of the native conformations of proteins. ${ }^{11,12}$ The sensitivity of disulphide metathesis to environmental stimuli has been employed in dynamic covalent chemistry (DCC) ${ }^{13-16}$ Contrary to traditional organic synthesis performed under kinetic regime, DCC involves thermodynamic control of reactions, which results in the spontaneous elimination of less stable products. Conversely, in kinetically controlled reactions, the form of substrates and catalysts, as well as reaction conditions, must be carefully chosen in order to favour the target products over the substrates and possible intermediates. ${ }^{13}$ In most reversible disulphide systems, the exchange reactions are proceed by a nucleophilic attack of a free thiolate at the disulphide bond and often require long equilibration times, even in the presence of a catalysts, which limits their applications. ${ }^{16}$ Only recently some significant progress towards cleaner and more efficient methods of disulphide metathesis was reported. ${ }^{17-22}$ A Brønsted basefree method requiring a phosphine to facilitate the reaction was described by Ramstrom et al. ${ }^{18}$ Belenguer, Friščić and Sanders used mechanochemistry for high-conversion disul- phide metathesis in the presence of 1,8-diazabicyclo[5.4.0] undec-7-ene. ${ }^{17,20}$ Pittelkow's group found that diselenides spontaneously exchange in water and can be used to catalyse disulphide exchange. ${ }^{21}$ Fritze and Delius showed that ultrasounds support the exchange of disulphides in $\mathrm{CH}_{3} \mathrm{Cl}^{19}$ Most recently, we have shown that high pressure can promote the metathesis between homodimeric bis(4chloroophenyl)disulphide with bis(2-nitrophenyl)disulphide without any catalyst or reducing agents and the heterodimeric product was obtained in several solvents. ${ }^{23}$

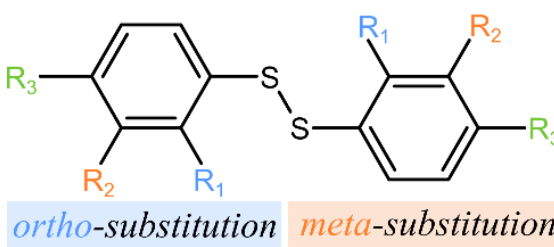

para-substitution

(9) $\mathrm{R}_{3}=\mathrm{CH}_{3}$

(10) $\mathrm{R}_{3}=\mathrm{Br}$

(11) $\mathrm{R}_{3}=\mathrm{OH}$

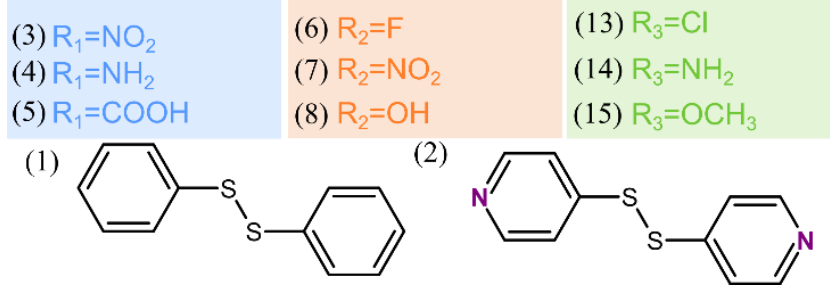

Figure 1. Aryl disulphides investigated under high-pressure. The colour code indicates molecules which are: (blue) orto-; (red) meta-; and (green) para-substituted. 
Presently, we have explored the mechanism of pressure-induced disulphides metathesis. For this purpose, we have performed in a diamond-anvil cell (DAC) 35 reactions between 15 homodimeric disulphides that differ in the character, size and position of their substituents. The effects of strongly electron withdrawing groups (EWG) $\mathrm{COOH}$ and $\mathrm{NO}_{2}$, electron donating groups (EDG) $\mathrm{NH}_{2}, \mathrm{OCH}_{3}, \mathrm{CH}_{3}, \mathrm{OH}$, as well as halides displaying dual positive-mesomeric and negativeinduction features $(\mathrm{Cl}, \mathrm{Br}$ and $\mathrm{F})$ have been studied and compared. Reactions were conducted in three different solvents: polar protic isopropanol and methanol, as well as polar aprotic acetonitrile. They remain liquid in the range of our highpressure experiments and secure the hydrostatic conditions. ${ }^{24,25}$ Due to low freezing pressure the nonpolar solvents were not considered for this experiments. Under pressure and without catalyst, we have obtained 21 different heterodimeric aryl disulphides. In six cases, after optimizing the reaction conditions, the products precipitate as single crystals and their structures could be determined by X-ray diffraction. The formation of 15 other products has been confirmed by mass spectrometry. Our results reveal the role of high-pressure reactions and their advantages for the DCC.

\section{RESULTS AND DISCUSSION}

It was established that high pressure increases the rate of reaction allowing it to run at lower temperatures. ${ }^{26-29}$ Among the most prominent reactions accelerated by high-pressure are Diels-Alder reactions, 1,3-dipolar cycloadditions, [2+2] cycloadditions, sigmatropic rearrangements and radical polymerizations. Although the impact of pressure on the reaction equilibrium is intuitive, it must be considered on several levels. At the molecular scale, when new bonds are formed, the reaction is accelerated by pressure due to reduced intermolecular distances and molecular volume. The reverse reaction, a homolytic bond cleavage, increase the volume and shifts the equilibrium toward the reactants. ${ }^{30}$ The compression, however, does not affect only the molecules themselves, but all reaction system changes its properties.

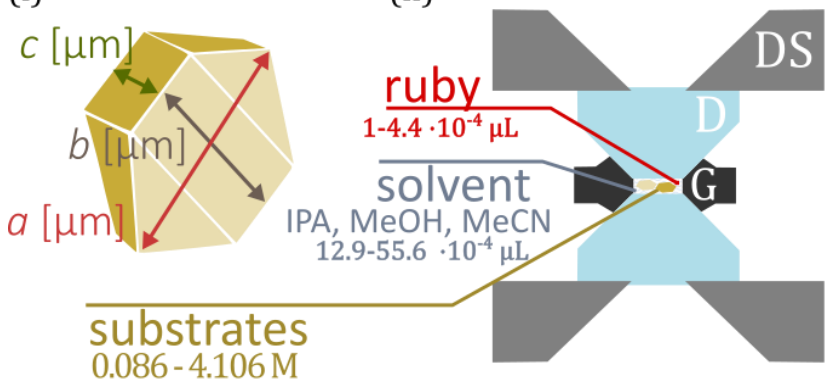

(i) $0.086-4.106 \mathrm{M}$
Under pressure the void spaces and distances between molecules are reduced, so is the space necessary for thermally induced motion and collisions. Additionally, the physical properties of the liquid environment are altered, resulting in higher boiling point, increased density, viscosity and the solubility of most compounds is reduced.

\section{Reaction procedure and optimization}

We performed a series of high-pressure reactions in a DAC, used as the high-pressure reactor. The DAC was modified by mounting the diamonds directly on the steel supports. ${ }^{31}$ All reactions were conducted according to the same procedure (Figure 2). Two homodimeric substrates in the form of single crystal were inspected through a microscope and their grains of equal $V_{s i} d_{i} / m_{s i}\left(V_{s i}, d_{i}\right.$ and $M_{s i}$ are the grain volume, density and molecular weight of substrate, respectively) were selected in order to obtain 1:1 molar ratio. Then the grains were loaded into the DAC chamber, together with small ruby spheres for pressure calibration..$^{32,33}$ Then the DAC chamber was filled with a solvent, sealed and compressed. The solvent volume $\left(V_{\text {sol }}\right)$ is:

$V_{\text {sol }}=V_{D A C}-\left[\left(V_{\mathrm{si}}+V_{\text {sii }}\right)+V_{r}\right]$,

were $V_{D A C}$ is the DAC chamber volume, and $V_{r}$ is the volume of the ruby sphere, and the molar concertation $\left(c_{\mathrm{i}}\right)$ of substrates is

$c_{i}=\left(V_{\mathrm{si}} \times d_{s i} / M_{s i}\right) / V_{\mathrm{sol}}$.

Then the DAC was heated until both reactants dissolved. Depending on the experiment - the substrates, their concertation, the solvent and pressure - the dissolution required the temperature from $323 \mathrm{~K}$ to $400 \mathrm{~K}$. After dissolving the substrates, the DAC was cooled down, and when the DAC chamber was unsealed the solvent evaporated. The crude solid precipitate was analyzed by a direct insertion probemass spectrometry (DIP-MS) and X-ray diffraction.

The DIP-MS does not require gas chromatographic separation, which eliminates the possibility of the reverse reaction in the analyzed product. High-temperatures used to sample ionization in DIP-MS technique, could be responsible for fragmentation of product/substrate molecules in the (iii)

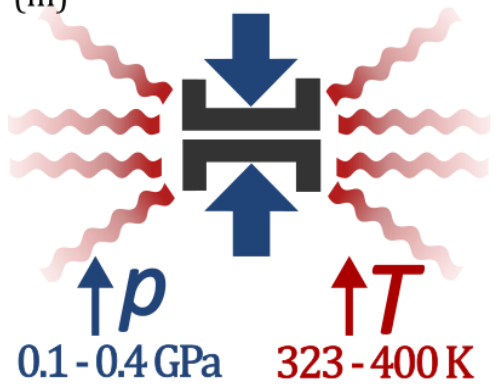

(iv)

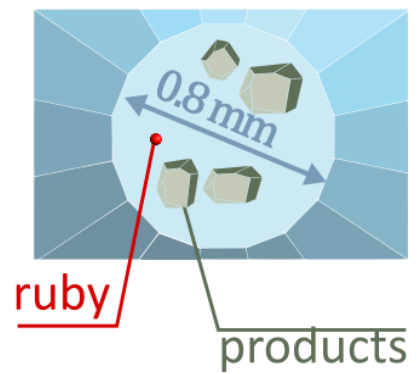

Figure 2. Schematic illustration of high-pressure reaction procedure: (i) single crystal measurements; (ii) loading the DAC chamber with two homodimeric substrates, together with ruby spheres, and filling the remaining volume with the solvent, 2-propanol (IPA), methanol $(\mathrm{MeOH})$ or acetonitrile $(\mathrm{MeCN})$, the DAC consists of two parallel diamonds (D) supported by steal discs (DS) and squeezed metal gasket (G); (iii) compression and heating of the DAC reactor till the dissolution of substrates; and (iv) recovering and analysis of the solid precipitate 
analysed sample; therefore, the DIP-MS was used mainly for quickly evaluating the yield. The reaction conditions were optimized by repeating the reaction several times with different concentrations, solvents, pressures and temperatures. For 6 out of 21 reactions (Sections 3 in SI), the yields approached $100 \%$ and precipitate in a form of single crystals of quality sufficiently high for X-ray structural study.

The crystals of heterodimeric products were recovered from the DAC chamber and analyzed on a SuperNova diffractometer equipped with a microfocus X-ray tube (detailed crystallographic data are summarized in Table S39, in Section 7 of Supporting Information). In order to establish the reaction equilibria in the solution for successfully exchanged systems $(11 / 14 ; 3 / 14 ; 9 / 13 ; 4 / 13 ; 4 / 12 ; 4 / 9 ; 4 / 7 ; 1 / 2 ;$ $10 / 12 ; 3 / 9 ; 3 / 10$ ), at ambient- and high-pressure, we have collected ${ }^{13} \mathrm{C}$-NMR spectra of homodimeric mixtures in 2propanol- $\mathrm{d} 8$ and $\mathrm{CD}_{3} \mathrm{CN}$ before and after compression to 0.4 $\mathrm{GPa}$ (for the experiment procedure look at 1.2.1 and for obtained spectra Section 5 of SI). Interestingly, the solubility of 3,12 and 5 in 2-propanol-d8 and $\mathrm{CD}_{3} \mathrm{CN}$ at ambient con-

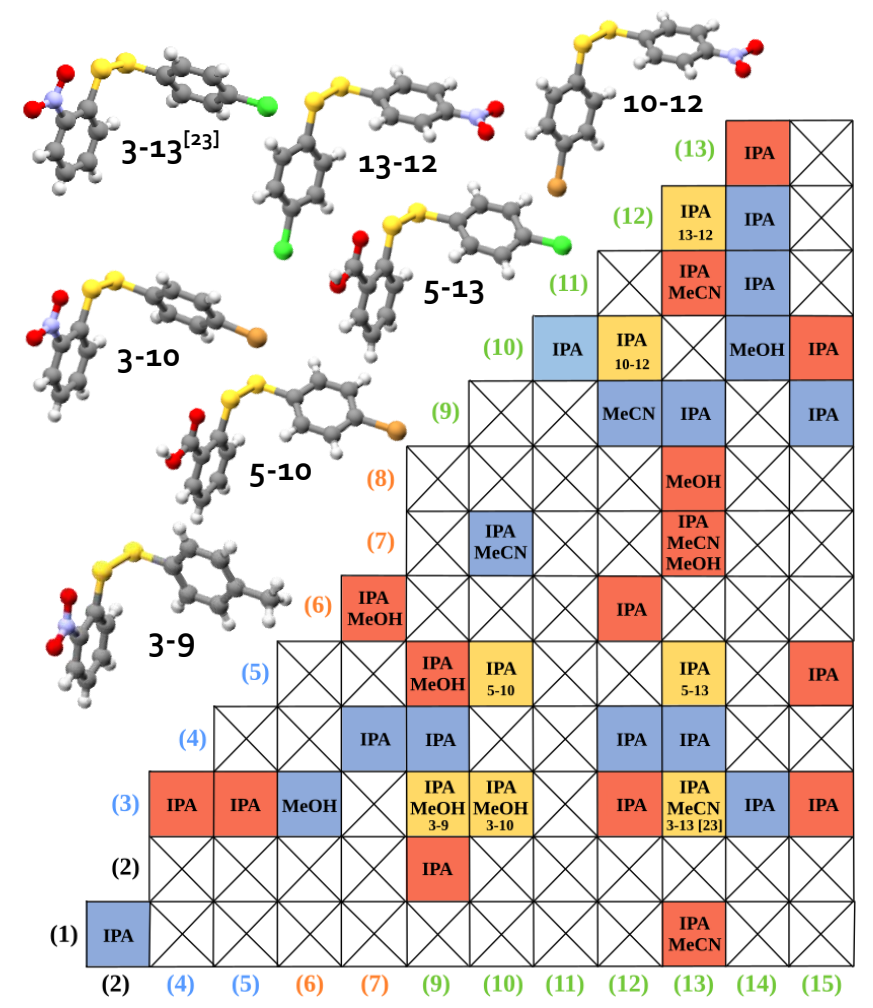

Figure 3. Compilation of all high-pressure reactions presently investigated; the numbers in brackets label to the substrates in Figure 1. Yellow colour mark the high-yield heterodimer products characterized by X-ray diffraction; blue - are products detected by DIP-MS spectra and red - no heterodimer traces. Crosses indicate that no reactions were performed. The

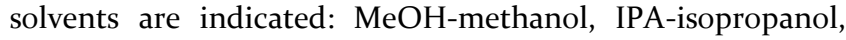
$\mathrm{MeCN}$-acetonitrile. The inset show heterodimers obtained in a form of single crystals. ditions was extremely poor (spectra 5.1.4 and 5.1.10 of Section 5 in SI). The ${ }^{13} \mathrm{C}-\mathrm{NMR}$ spectrum of $\mathbf{5}$, could be collected only in DMSO-d6 (5.1.12 of SI).

Additionally, the effect of high-temperature on solid homodimeric mixture during slow heating to $673 \mathrm{~K}$ was investigated by TG-DSC. No thermal effects associated with chemical reaction were detected (Sections 6.1 in SI). The influence of anisotropic pressure on the mixtures of solid homodimers with and without a few drops (4-5 $\mu \mathrm{L})$ of IPA, investigated by powder X-ray diffraction showed no traces of heterodimeric products (Sections 6.2 in SI).

\section{High-pressure chemistry}

The control over the high-pressure reactions, as at ambient conditions, can be achieved by two different basic approaches. In a kinetically controlled reaction, the differences between the volume of activation $V^{\ddagger}$, which according to transition state theory, is a difference between the partial molar volumes of the transition state and the sums of the partial volumes of the reactants at the same temperature and pressure; leading to the different product molecules must be achieved. Alteration of $V^{\neq}$, can be achieved either by a pressure-induced change of reaction mechanism or by the difference between the volumes of the transition structures within the same, or at least similar, mechanism. In a thermodynamically controlled reaction, reaction rate increases when reaction volume, $V^{\mathrm{o}}$, decreases. Recently, it was shown both theoretically and experimentally, that instead of proposed before $[2+2]$ metathesis, the S-S reversible bond cleavage and formation, is radical-mediated (Scheme 1). ${ }^{34-38}$

\section{Scheme 1. Mechanisms of an $[2+1]$ radical mediated di- sulphide exchange}

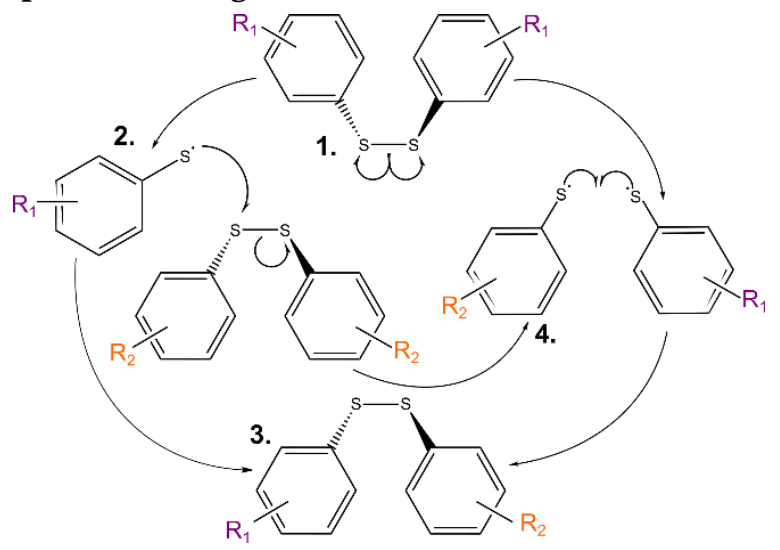

According to this mechanism, the reaction initiates when sulfenyl radicals are formed (step 1). Bond dissociation is a process associated with a volume expansion and at highpressure, it is a rate determining step which could possibly even lead to reaction inhibition. Thus it is apparent that at high-pressure this process does not resemble the UV or ultrasounds generation of radicals but rather a chain reaction 
where a positive feedback leads to a self-amplifying chain of events.

The formation of sulfenyl radicals strongly depends on S-S bond energy. Theoretical studies on disulphide based polymers connect the S-S length with bond energy in an inverse corelation. ${ }^{37}$ Our survey of the molecular structures of homodimeric aryl disulphides reported in this work and those deposited in the Cambridge Structural Database (CSD Version 5.41, ConQuest Version 2.05, Figure S1) show that the substitution of EDG in para- or orto- or EWG in ortoposition, as well as the presence of a heteroatom in the aromatic ring, result in longer S-S distances. It appears that a substitution by the EDG adds electron density to a conjugated $\pi$-system via resonance or inductive effects. This increase in electron density into the antibonding $\sigma_{\mathrm{SS}}{ }^{*}$ bond, followed by elongation of the S-S bond, lowers its dissociation energy. Similarly, the ortho-positioned EWG, due to the resonance effect, creates an electron-deficient $(\delta+)$ region at the S-atom and weakens the S-S bond. In contrast, para- substituted EWG the opposite effect, shortening the S-S bond can be observed. At high-pressures, the energy necessary for bond dissociation can be achieved at lower temperatures, as it was shown for $9 .{ }^{39}$ Its recrystallization at $0.45 \mathrm{GPa}$ result in $\mathrm{S}-\mathrm{S}$ bond elongation from $2.024 \AA$, at ambient conditions, to $2.058 \AA$ connected to a phase transition. ${ }^{39}$

Once free radicals are generated, they attack other homodimers and produce new free radicals (step 2), along with a product molecule (step 3). At this stage, the reverse recombinations are also possible. Although at high-pressure the reverse reaction can only occur if formation of product would lead to negligible reduction of $V^{0}$. The reaction terminates when two different radical species react with each other to form a heterodimeric adduct (step 4).

In order to resolve if the pressure reactions in DAC chamber (conducted in the solid-solute-solid sequence, cf. section: Reaction procedure and optimization) are proceed under thermodynamic or kinetic regime i.e. the reactions are generally characterized by negative $V^{\mathrm{o}}$ or there is some kinetic aspect involved, we compared the results with the pressure effect on compressed mixtures of homodimers dissolved in 2-propanol-d8 and $\mathrm{CD}_{3} \mathrm{CN}$. For equilibrium reactions with large negative $V^{\circ}$, the application of high-pressure would lead to a significant yield increasion. This relationship comes from the fact that a small change in free Gibbs energy $\left(\Delta G^{o}\right)$ leads to a logarithmically amplified change in equilibrium position, according to;

$\Delta G^{o}=\partial \ln K / \partial p T=-\Delta V^{\mathrm{o}} / \mathrm{R} T$,

where $\mathrm{R}$ is the ideal gas constant, $T$ temperature and $K$ the equilibrium constant.

A kinetic study has been performed for 11 different systems, corresponding to reactions successfully conducted in the DAC. Homodimers were first dissolved in 2-propanol-d8 and $\mathrm{CD}_{3} \mathrm{CN}$, then mixed and after $12 \mathrm{~h}$ in ambient conditions, their ${ }^{13} \mathrm{C}$-NMR spectra were collected. In seven systems
$(11 / 14 ; 9 / 13 ; 3 / 14 ; 10 / 12 ; 3 / 9 ; \underline{3 / 10} ; 4 / 12)$ no product was observed, while in four other reactions $(4 / 9 ; 4 / 7 ; 1 / 2 ; 4 / 13)$ an equilibrium established below $50 \%$ conversion to the product. Next, these same systems were compressed isothermally at $0.4 \mathrm{GPa}$ and their spectra were collected within a $0.5 \mathrm{~h}$ after the pressure was released. Surprisingly, compression of the dissolved homodimers, in reactions $11 / 14 ; \mathbf{3 / 1 4}$; $10 / 12 ; 3 / 9 ; 3 / 10 ; 4 / 12$ still did not result in formation of the heterodimeric product (cf. SI). Amount of formed product for $11 / 14 ; 3 / 14 ; 9 / 13 ; 4 / 13$ and $3 / 9$, was additionally confirmed by DIP-MS (Section 5.5 of SI). We have connected these results with the intrinsic volume profiles of the reactions (Figure 4), based on van der Waals models of substrate and product molecules.

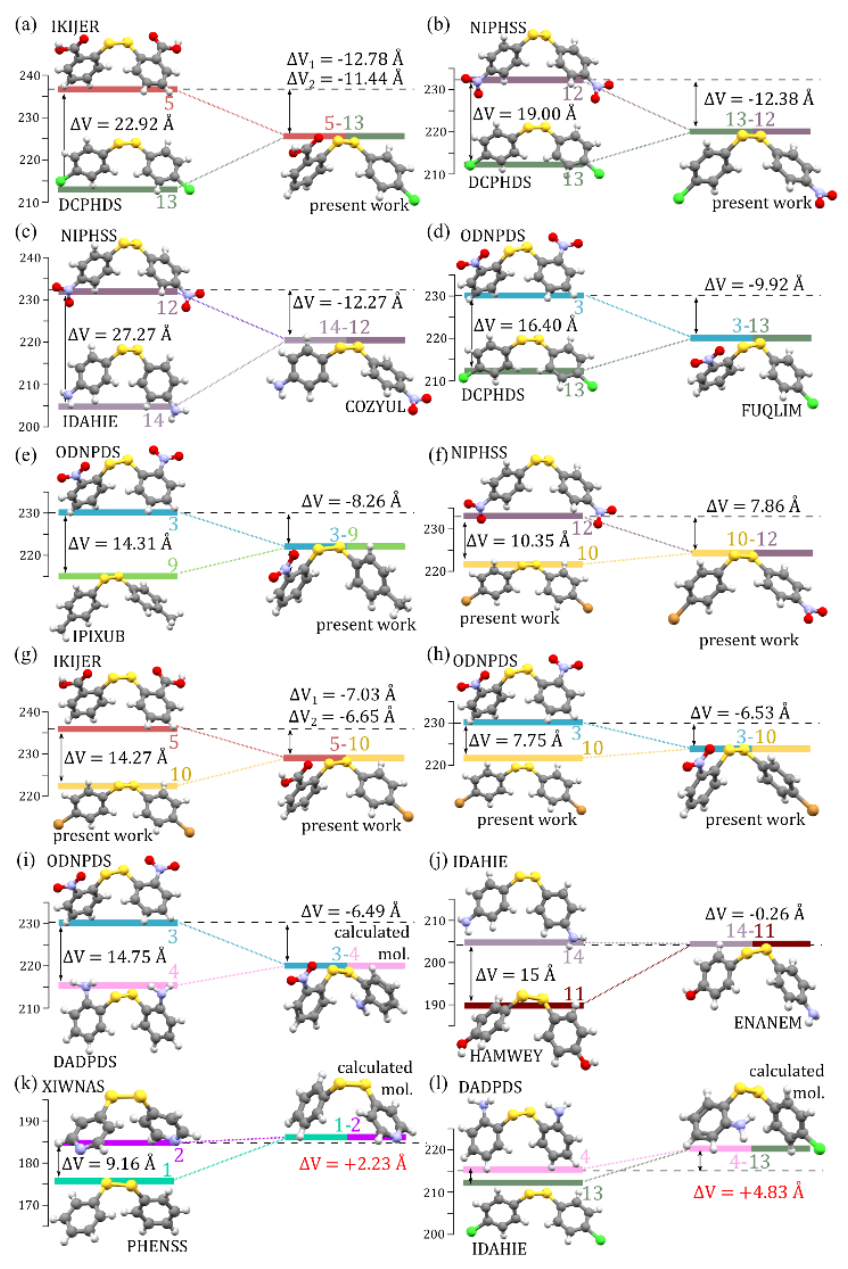

Figure 4. Van der Waals volumes profile calculated for the exchange reaction. The structures were determined in this work, or retrieved from the CSD (where six-letter refcodes are given). For reactions $\mathbf{3 / 4}, \mathbf{1} / \mathbf{2}, 4 / \mathbf{1 3}$ the molecular conformation of product molecule has been calculated in program Gaussiani6 with B3LYP/6-311+ G (2d, 2p) level of theory. 
Owe to significantly different volume of substituents $R_{\mathrm{i}}$ in substrates (Figure 1), the molecular volume of the heterodimer is close to the intermediate of the volumes of homodimers. Subtle departures from the mean value $\left(\Delta V_{m}\right)$ are mainly due to the $\mathrm{S}-\mathrm{S}$ bond length changes $(\Delta L)$, according to approximate formula $\Delta V_{m} \approx 18.3 \Delta L\left[\AA^{3}\right]$. The $S-S$ length change of $0.07 \AA$, e.g. between 2.01 and $2.07 \AA$, corresponds to $\Delta V_{m}=1.0 \AA^{3}$ comparable to the accuracy of our volume calculations. Surprisingly, two systems 1/2, 4/13 with the biggest positive $\Delta V^{\mathrm{o}}\left(+2.23 \AA^{3}\right.$ and $+4.83 \AA^{3}$, respectively) the mixtures at ambient conditions equilibrate with approx. $1: 1$ products to substrates, while reactions $\mathbf{3 / 9}, \mathbf{1 0} / \mathbf{1 2}, \mathbf{3 / 1 0}$, $14 / 11$ characterized by $\Delta V^{\mathrm{o}}$ reduction, did not generate any products even after compression. It is apparent that compression of $\mathbf{1 / 2}, \mathbf{4 / 1 3}$ will, and is, not resulting in reaction acceleration. For the systems $\mathbf{3 / 9}, \mathbf{1 0} / \mathbf{1 2}, \mathbf{3} / \mathbf{1 0}, \mathbf{1 4} / \mathbf{1 1}$, however, where the $\Delta V^{0}$ decreases, and in system $\mathbf{3 / 9}$ is equal to -8.26 $\AA^{3}$ is not enough to compensate the $\Delta V^{\mathrm{o}}$ gained while radicals were formed and thus are inhibited.

The molecular conformation is another variable capable of stimulating the heterodimers formation at highpressure. ${ }^{40}$ The intermolecular interactions can considerably change soft conformational parameters and may be relevant for improving the reactivity of disulphides. ${ }^{22,41}$ It was suggested for free radical polymerization of ethylene that the formation of various supramolecular intermediate forms can be unique for high-pressure conditions and thus essential for the increase in conversion to the product form. ${ }^{42}$ In all heterodimeric disulphides the $\mathrm{C}-\mathrm{S}-\mathrm{S}-\mathrm{C}$ torsional angles are all within $\pm\left(85^{\circ} \pm 3^{\circ}\right)$. The value of this torsion angle is dominated by $3 p$ lone pairs on the two $\mathrm{S}$ atoms, which minimize their mutual overlap and repulsion integrals for the orthogonal position. ${ }^{43}$

\section{Control by the Entropy}

The synthesis of heterodimers $(\mathrm{AB})$ from homodimers $\left(\mathrm{A}_{2}\right.$ and $\mathrm{B}_{2}$ ) can be achieved, even with $100 \%$ yield, in the entropy-driven kinetic process schematically depicted in Figure 5. The process consists of several stages, which initially maximizes the entropy $(S)$ of the system and then triggers the crystallization of the heterodimer. High pressure plays an essential role, allowing the entropy to increase beyond that attainable at atmospheric pressure. Thus, at stage $\mathbf{1}$ this system consisting of equimolar amounts of homodimers $\mathrm{A}_{2}$ and $\mathrm{B}_{2}$ and some solvent $(\mathrm{F})$ is closed in a DAC chamber. The chamber is sealed and pressurized to about $200 \mathrm{MPa}(2000$ bar). This adds the compression work component to the Gibbs free energy $(G)$ and most of homodimers $\mathrm{A}_{2}$ and $\mathrm{B}_{2}$ remain in crystalline form. The solubility usually decreases with increasing pressure, so apart from the work contribution $(p V)$ the entropy $(S)$ decreases due to a smaller portion of the crystals dissolved at stage $\mathbf{2}$ compared to stage $\mathbf{1}$. In stage $\mathbf{3}$, the sample is heated up until stage $\mathbf{4}$, when crystals $A_{2}$ and $\mathrm{B}_{2}$ dissolve. At this point, the system assumes the maximum entropy when molecules $\mathrm{A}_{2}, \mathrm{~B}_{2}$ and $\mathrm{F}$ are randomly mixed. At this stage the dimers still increase entropy through their environmental variation. Accordingly, to the experimental reports, and also to the theoretical calculations, at high pressure the S-S distance significantly increase facilitating the bond cleavage; also the disulphide conformation can change and become more 'open' to be attacked by sulfenyl radical. $^{22,41,44}$ Then the entropy can be further increased at still higher temperature when the homodimers start to dissociate into radicals at stage $\mathbf{5}$. The increased number of radicals intensifies the chain reactions, leading to heterodimers $A B$, whereas some recombinations to $\mathrm{A}_{2}$ and $\mathrm{B}_{2}$ when the product and substrate are both comparable in volume. On lowering the temperature, the system (stage 6) tends to reduce the entropy while the ratio between the homo- and heterodimers depends on the equilibrium in the solution at given thermodynamic conditions. At stage 7, a significant reduction of entropy is achieved by the process of nucleation.

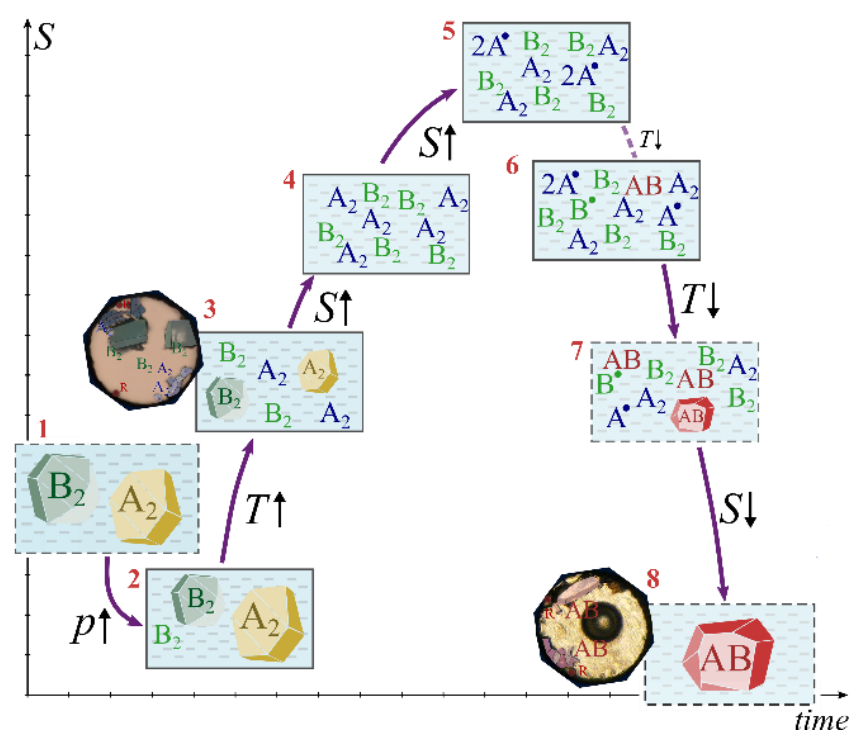

Figure 5. Schematic illustration of the entropy driven synthesis of heterodimers $\mathrm{AB}$ from homodimers $\mathrm{A}_{2}$ and $\mathrm{B}_{2}$ dissolved in solvent (grey dashes). The stages of the process (red numbers) are described in the text. The open and closed systems has been indicated by the dashed and solid edges of boxes, respectively.

It is characteristic of high temperature that particles are well mixed, as the grouping of identical particles would decrease the entropy. This strongly favours the nucleation of heterodimers. At stage 7 , the solubility of $A_{2}, B_{2}$ and $A B$ in solvent is very important as the low solubility of heterodimer $A B$ increases its chances to nucleate. The kinetic crystallization of $\mathrm{AB}$ shifts the reaction equilibrium toward the product side and fuels the synthesis of heterodimers AB. At the final stage $\mathbf{8}$, at $296 \mathrm{~K}$, all homodimers reacted into heterodimers are present in the solid form of one or more solid grains except for the small numbers of $A_{2}, B_{2}$ and $A B$ dimers (hence low entropy $S$ ) remaining dissolved in solution. Kinetic crystallization following the reduction of temperature strongly reduces the entropy because just on substance is ordered in the 
crystal. It is apparent that the solubility of $\mathrm{AB}$ compared to those of $A_{2}$ and $B_{2}$ is important for nucleation. It is reasonable to assume that relative to homodimers, the polarity of heterodimeric molecules significantly increases, which in turn increases the electrostatic cohesion forces and reduces the crystals solubility.

It should be stressed, that high-pressure is used as an efficient method of sealing the system. For a closed system, the entropy can be increased to the required level (stage 5) of strongly mixed molecules and radicals. After stage 7, the confining role of the DAC chamber is continued, irrespective of the pressure value, which can be kept high or released.

\section{CONCLUSIONS}

Disulphide exchange reactions have been systematically investigated for a series of representative, and in some cases unique, aryl disulphides with distinct chemical features, in different environments and in extreme conditions of highpressure and high-temperature. These results provide a microscopic insight into non-catalytic disulphide exchange reactions. The application of high-pressure extends the thermodynamic conditions to high temperature not attainable in 'open' systems. Owing to the confined reaction space, the entropy of the system can be increased in a controlled manner to the stage, when the substrates are dissolved at high concentrations, well mixed, ant their molecules excited into high $E_{p}$ conformers, high vibrational states and dissociated. Then by lowering temperature the high-entropy nucleation and subsequent kinetic crystallization can overcome the thermodynamic equilibrium and result in the high yields of exchanged disulphides without catalyst required for ambient-pressure reactions. The comprehensive studies, utilizing DIP-MS, NMR, TG-DSC, single-crystal and powder diffraction and theoretical calculations, corroborate this mechanism and understanding of the disulphide exchange reactions. The application of high-pressure is essential for the high-entropy effects (including the excited conformational states, S-S bond cleavage), the nucleation and subsequent kinetic crystallization, which are the key elements leading to the non-catalytic disulphide exchange. These results indicate the high-pressure techniques as most suited for tailoring a one-pot, high-yield reaction systems leading to high-quality single crystalline products. It can be noted that the pressure of about $0.2 \mathrm{GPa}$ proved sufficient for sealing the reaction space is technologically well accessible in various types of equipment, for example in the lower rang of operation of many piston-and-cylinder devices and in many autoclaves. At the same time the high-entropy methods eliminate the catalyst, which apart from the environmental issues including further purification of obtained product, make them financially competitive.

\section{ASSOCIATED CONTENT}

In detail description of all experimental data, including highpressure equipment, used analytic methods, volume calculations, as well as the summarized results, with all NMR and DIP-MS spectra, of all conducted high-pressure reactions in DAC and in compressed-mixtures reactions, description regarding deconvolution of the accompanying effect of temperature and non-hydrostatic pressure acting alone, details about molecular volume calculations and tabularized results, detailed crystallographic data of novel substrates and products and synthetic procedure for $\mathbf{1 0}$ is available free of charge via the Internet at http://pubs.acs.org."

The CIF files have been deposited in Cambridge Structural Database (CCDC, www.ccdc.cam.ac.uk) as supplementary publications with reference numbers 2033598-2033603 for synthesized heterodimers 3-10, 10-12, 13-12, 3-9, 5-13, 5-10 and 2033604-2033606 for 15, 6 and 10, respectively.

\section{AUTHOR INFORMATION}

\section{Corresponding Author}

*szymon.sobczak@amu.edu.pl

*katran@amu.edu.pl

\section{Author Contributions}

All authors have given approval to the final version of the manuscript.

\section{Funding Sources}

We thank the Polish National Science Centre (grant PRELUDIUM 2017/27/N/ST5/00693)

\section{ACKNOWLEDGMENT}

The Authors are grateful to Dominika Czerwonka of Department of Chemistry, Adam Mickiewicz University, Poznan; Jean-Louis Do of Department of Chemistry, McGill University, and Michal Andrzejewski of Department of Chemistry and Biochemistry, University of Bern, for valuable suggestions and fruitful discussions.

\section{ABBREVIATIONS}

DCC, dynamic covalent chemistry; DAC, diamond anvil cell; EWG, EDG electron donating or withdrawing group; DIP-MS, direct insertion probe - mass spectrometry.

\section{REFERENCES}

(1) Forbes, E. S. The Load-Carrying Action of OrganoSulphur Compounds-A Review. Wear 1970, 15 (2), 8796. https://doi.org/10.1016/0043-1648(70)90002-5.

(2) ALlUM, K. G.; Forbes, E. S. The Load-Carrying Properties of Organic Sulpher Compounds. Part II. The Influence of Chemical Structure on the Anti-Wear Properties of Organic Disulphides. J. Inst. Pet. 1967, 53 (521), 174

(3) Yanez, C. O.; Andrade, C. D.; Belfield, K. D. Characterization of Novel Sulfonium Photoacid Generators and Their Microwave-Assisted Synthesis. Chem. Commun. 2009, No. 7, 827-829. https://doi.org/10.1039/b815831b. 
Labelle, M.; Belley, M.; Gareau, Y.; Gauthier, J. Y.; Guay, D.; Gordon, R.; Grossman, S. G.; Jones, T. R.; Leblanc, Y.; McAuliffe, M.; et al. Discovery of MK-0476, a Potent and Orally Active Leukotriene D4 Receptor Antagonist Devoid of Peroxisomal Enxyme Induction. Bioorganic Med. Chem. Lett. 1995. https://doi.org/10.1016/0960894X(95)00023-M.

(5) Wang, Y.; Chackalamannil, S.; Hu, Z.; Clader, J. W.; Greenlee, W.; Billard, W.; Binch, H.; Crosby, G.; Ruperto, V.; Duffy, R. A.; et al. Design and Synthesis of Piperidinyl Piperidine Analogues as Potent and Selective M2 Muscarinic Receptor Antagonists. Bioorganic Med. Chem. Lett. 2000, 10 (20), 2247-2250. https://doi.org/10.1016/S0960-894X(00)00457-1.

Ram, V. J.; Vlietinck, A. J. Chemotherapeutical Agents. VII. Synthesis and Pesticidal Activities of Sulphides and Sulphones Derived from Bis[4-Aryl-1,2,4-Triazoline-5Thione-3-Yl]Alkane and 5-Phenyl-1,3,4-Oxadiazole-2Thione. J. Heterocycl. Chem. 1988, 25 (1), 253-256. https://doi.org/10.1002/jhet.5570250141.

Otzen, T.; Wempe, E. G.; Kunz, B.; Bartels, R.; LehwarkYvetot, G.; Hänsel, W.; Schaper, K. J.; Seydel, J. K. Folate-Synthesizing Enzyme System as Target for Development of Inhibitors and Inhibitor Combinations against Candida Albicans - Synthesis and Biological Activity of New 2,4-Diaminopyrimidines and 4'Substituted 4-Aminodiphenyl Sulfones. J. Med. Chem. 2004, 47 (1), 240-253. https://doi.org/10.1021/jm030931w.

(8) Alcaraz, M. L.; Atkinson, S.; Cornwall, P.; Foster, A. C.; Gill, D. M.; Humphries, L. A.; Keegan, P. S.; Kemp, R.; Merifield, E.; Nixon, R. A.; et al. Efficient Syntheses of AZD4407 via Thioether Formation by Nucleophilic Attack of Organometallic Species on Sulphur. Org. Process Res. Dev. 2005, 9 (5), 555-569. https://doi.org/10.1021/op0500483.

(9) Llauger, L.; He, H.; Kim, J.; Aguirre, J.; Rosen, N.; Peters, U.; Davies, P.; Chiosis, G. Evaluation of 8-Arylsulfanyl, 8-Arylsulfoxyl, and 8-Arylsulfonyl Adenine Derivatives as Inhibitors of the Heat Shock Protein 90. J. Med. Chem. 2005, 48 (8), 2892-2905. https://doi.org/10.1021/jm049012b.

(10) Pasquini, S.; Mugnaini, C.; Tintori, C.; Botta, M.; Trejos, A.; Arvela, R. K.; Larhed, M.; Witvrouw, M.; Michiels, M.; Christ, F.; et al. Investigations on the 4-Quinolone-3Carboxylic Acid Motif. 1. Synthesis and StructureActivity Relationship of a Class of Human Immunodeficiency Virus Type 1 Integrase Inhibitors. $J$. Med. Chem. 2008, 51 (16), 5125-5129. https://doi.org/10.1021/jm8003784.

(11) Ladenstein, R.; Ren, B. Reconsideration of an Early Dogma, Saying 'There Is No Evidence for Disulfide Bonds in Proteins from Archaea'. Extremophiles 2008, 12 (1), 29-38. https://doi.org/10.1007/s00792-007-0076-z.

(12) Sevier, C. S.; Kaiser, C. A. Formation and Transfer of Disulphide Bonds in Living Cells. Nat. Rev. Mol. Cell Biol. 2002, 3 (11), 836-847. https://doi.org/10.1038/nrm954.

(13) Gasparini, G.; Dal Molin, M.; Lovato, A.; Prins, L. J. Dynamic Covalent Chemistry. Supramol. Chem. 2012, No. Dcc. https://doi.org/10.1002/9780470661345.smc161.

(14) Jin, Y.; Yu, C.; Denman, R. J.; Zhang, W. Recent Advances in Dynamic Covalent Chemistry. Chem. Soc.
Rev. 2013, $42 \quad$ (16) $6634-6654$ https://doi.org/10.1039/c3cs60044k.

(15) Corbett, P. T.; Leclaire, J.; Vial, L.; West, K. R.; Wietor, J. L.; Sanders, J. K. M.; Otto, S. Dynamic Combinatorial Chemistry. Chem. Rev. 2006, 106 (9), 3652-3711. https://doi.org/10.1021/cr020452p.

(16) Black, S. P.; Sanders, J. K. M.; Stefankiewicz, A. R. Disulfide Exchange: Exposing Supramolecular Reactivity through Dynamic Covalent Chemistry. Chem. Soc. Rev. 2014, $43 \quad$ (6), 1861-1872. https://doi.org/10.1039/c3cs60326a.

(17) Belenguer, A. M.; Lampronti, G. I.; Wales, D. J.; Sanders, J. K. M. Direct Observation of Intermediates in a Thermodynamically Controlled Solid-State Dynamic Covalent Reaction. J. Am. Chem. Soc. 2014, 136 (46), 16156-16166. https://doi.org/10.1021/ja500707z.

(18) Caraballo, R.; Rahm, M.; Vongvilai, P.; Brinck, T.; Ramström, O. Phosphine-Catalyzed Disulfide Metathesis. Chem. Commun. 2008, No. 48, 6603-6605. https://doi.org/10.1039/b815710c.

(19) Fritze, U. F.; Von Delius, M. Dynamic Disulfide Metathesis Induced by Ultrasound. Chem. Commun. 2016, 52 (38), 6363-6366. https://doi.org/10.1039/c6cc02034h.

(20) Belenguer, A. M.; Friščić, T.; Day, G. M.; Sanders, J. K M. Solid-State Dynamic Combinatorial Chemistry: Reversibility and Thermodynamic Product Selection in Covalent Mechanosynthesis. Chem. Sci. 2011, 2 (4), 696. https://doi.org/10.1039/c0sc00533a.

(21) Rasmussen, B.; Sørensen, A.; Gotfredsen, H.; Pittelkow, M. Dynamic Combinatorial Chemistry with Diselenides and Disulfides in Water. Chem. Commun. 2014, 50 (28), 3716-3718. https://doi.org/10.1039/c4cc00523f.

(22) Dopieralski, P.; Ribas-Arino, J.; Anjukandi, P.; Krupicka, M.; Kiss, J.; Marx, D. The Janus-Faced Role of External Forces in Mechanochemical Disulfide Bond Cleavage. Nat. Chem. 2013, 5 (8), 685-691. https://doi.org/10.1038/nchem.1676.

(23) Sobczak, S.; Drożdż, W.; Lampronti, G. I.; Belenguer, A. M. A.; Katrusiak, A.; Stefankiewicz, A. R. Dynamic Covalent Chemistry under High-Pressure:A New Route to Disulfide Metathesis. Chem. - A Eur. J. 2018, 24 (35), 8769-8773. https://doi.org/10.1002/chem.201801740.

(24) Klotz, S.; Chervin, J. C.; Munsch, P.; Le Marchand, G. Hydrostatic Limits of 11 Pressure Transmitting Media. $J$. Phys. D. Appl. Phys. 2009, 42 (7). https://doi.org/10.1088/0022-3727/42/7/075413.

(25) Olejniczak, A.; Katrusiak, A. Supramolecular Reaction between Pressure-Frozen Acetonitrile Phases $\alpha$ and $\beta$. $J$. Phys. Chem. B 2008, 112 (24), 7183-7190. https://doi.org/10.1021/jp800753n.

(26) van Eldik, R.; Klärner, F. G. High Pressure Chemistry: Synthetic, Mechanistic, and Supercritical Applications; van Eldik, R., Klärner, F., Eds.; Wiley, 2007. https://doi.org/10.1002/9783527612628.

(27) Benson, S. W.; Berson, J. A. The Effect of Pressure on the Rate and Equilibrium Constants of Chemical Reactions. The Calculation of Activation Volumes by Application of the Tait Equation. J. Am. Chem. Soc. 1962, 84 (2), 152158. https://doi.org/10.1021/ja00861a005.

(28) Asano, T.; Noble, W. J. L. Activation and Reaction Volumes in Solution. Chem. Rev. 1978, 78 (4), 407-489. https://doi.org/10.1021/cr60314a004.

(29) Noble, W. J. Organic Synthesis at High Pressure. In High Pressure Chemistry and Biochemistry; 1987. 
https://doi.org/10.1007/978-94-009-3827-4_12.

(30) van Eldik, R.; Klärner, F. G. High Pressure Chemistry: Synthetic, Mechanistic, and Supercritical Applications; Wiley-VCH, 2007. https://doi.org/10.1002/9783527612628.

(31) Merrill, L.; Bassett, W. A. Miniature Diamond Anvil Pressure Cell for Single Crystal X-Ray Diffraction Studies. Rev. Sci. Instrum. 1974, 45 (2), 290-294. https://doi.org/10.1063/1.1686607.

(32) Piermarini, G. J.; Block, S.; Barnett, J. D.; Forman, R. A. Calibration of the Pressure Dependence of the R1ruby Fluorescence Line to $195 \mathrm{Kbar}$. J. Appl. Phys. 1975, 46 (6), 2774-2780. https://doi.org/10.1063/1.321957.

(33) Mao, H. K.; Xu, J.; Bell, P. M. Calibration of the Ruby Pressure Gauge to 800 Kbar under Quasi-Hydrostatic Conditions. Journal of Geophysical Research. 1986, pp 4673-4676. https://doi.org/10.1029/jb091ib05p04673.

(34) Azcune, I.; Odriozola, I. Aromatic Disulfide Crosslinks in Polymer Systems: Self-Healing, Reprocessability, Recyclability and More. Eur. Polym. J. 2016, 84, 147160. https://doi.org/10.1016/j.eurpolymj.2016.09.023.

(35) Nevejans, S.; Ballard, N.; Miranda, J. I.; Reck, B.; Asua, J. M. The Underlying Mechanisms for Self-Healing of Poly(Disulfide)S. Phys. Chem. Chem. Phys. 2016, 18 (39), 27577-27583. https://doi.org/10.1039/c6cp04028d.

(36) Formoso, E.; Asua, J. M.; Matxain, J. M.; Ruipérez, F. The Role of Non-Covalent Interactions in the Self-Healing Mechanism of Disulfide-Based Polymers. Phys. Chem. Chem. Phys. 2017, 19 (28), 18461-18470. https://doi.org/10.1039/c7cp03570e.

(37) Matxain, J. M.; Asua, J. M.; Ruipérez, F. Design of New Disulfide-Based Organic Compounds for the Improvement of Self-Healing Materials. Phys. Chem. Chem. Phys. 2016, 18 (3), 1758-1770. https://doi.org/10.1039/c5cp06660c.

(38) Irigoyen, M.; Matxain, J. M.; Ruipérez, F. Effect of Molecular Structure in the Chain Mobility of Dichalcogenide-Based Polymers with Self-Healing Capacity. Polymers (Basel). 2019, $11 \quad$ (12). https://doi.org/10.3390/polym11121960.

(39) Sobczak, S.; Katrusiak, A. Colossal Strain Release by Conformational Energy Up-Conversion in a Compressed Molecular Crystal. J. Phys. Chem. C 2017, 121 (5), 25392545. https://doi.org/10.1021/acs.jpcc.6b11030.

(40) Structure Correlation; Bürgi, H., Dunitz, J. D., Eds.; Wiley, 1994. https://doi.org/10.1002/9783527616091.

(41) Dopieralski, P.; Ribas-Arino, J.; Anjukandi, P.; Krupicka, M.; Marx, D. Unexpected Mechanochemical Complexity in the Mechanistic Scenarios of Disulfide Bond Reduction in Alkaline Solution. Nat. Chem. 2017, 9 (2), 164-170. https://doi.org/10.1038/NCHEM.2632.

(42) Stoiljkovich, D.; Jovanovich, S. Mechanism of the HighPressure Free Radical Polymerization of Ethylene. J. Polym. Sci. A1. 1981, 19 (3), 741-747.

(43) Glidewell, C.; Low, J. N.; Wardell, J. L. Conformational Preferences and Supramolecular Aggregation in 2Nitrophenylthiolates: Disulfides and Thiosulfonates. Acta Crystallogr. Sect. B Struct. Sci. 2000, 56 (5), 893-905. https://doi.org/10.1107/S0108768100007114.

(44) Sobczak, S.; Katrusiak, A. Colossal Strain Release by Conformational Energy Up-Conversion in a Compressed Molecular Crystal. J. Phys. Chem. C 2017, 121 (5), 2539 2545. https://doi.org/10.1021/acs.jpcc.6b11030. 